\title{
Images in urology: Localized primary amyloidosis of the urinary bladder
}

Shawn Varghese; Nathan Colin Wong; Bobby Shayegan

McMaster University, Hamilton, ON, Canada

Cite as: Can Urol Assoc J 2017 Dec 1; Epub ahead of print. http://dx.doi.org/10.5489/cuaj.4825

Published online December 1, 2017

$* * *$

Amyloidosis is the extracellular deposition of amyloid fibrils into tissue, perhaps impairing regular organ functionality. Primary amyloidosis of the bladder is rare, affecting both genders equally between the ages of 40-80, with approximately 200 reported cases in the available literature. ${ }^{1}$ Although the exact mechanism of amyloidosis of the bladder is unknown, it is thought to be a result of a chronic or recurrent mucosal and submucosal inflammation. ${ }^{1}$ Common presentations include gross painless hematuria, lower urinary tract symptoms, and bladder mass found on imaging. As there are no specific manifestations and presentation often mimics bladder cancer, it may be commonly misdiagnosed and undergo possible overtreatment.

A 63-year-old man with long standing lower urinary tract symptoms was referred to our tertiary hospital for the evaluation of recurrent gross painless hematuria. He had a past medical history of hypercholesterolemia, hypertension and cardiac arrhythmia. Due to his lower urinary tract symptoms, he was previously diagnosed with benign prostatic hyperplasia and treated unsuccessfully with medical therapy. He had no history of underlying chronic inflammatory conditions. At a peripheral hospital, he underwent previous cystoscopy which revealed abnormal areas of the bladder mucosa along the right anterolateral wall suspicious for bladder cancer. However, subsequent biopsies were negative for malignancy. Computed tomography with intravenous contrast showed evidence of diffuse bladder thickening, more pronounced on the right side.

At our centre, he underwent repeat cystoscopy and complete transurethral resection of the persistent bladder lesions along the right anterolateral wall of the bladder (Figure 1A). Although the lesions were suspicious for bladder cancer, we conferred with our genitourinary pathologists regarding other diagnoses. Staining with Hematoxylin and eosin showed evidence of eosinophilic amorphous deposits (Figure 2B). Histopathology with Congo red showed positive green-apple birefringence under polarized light, consistent with amyloid deposition and a diagnosis of amyloidosis of the urinary bladder (Figure 2A).

Before a diagnosis of localized amyloidosis is made, it is imperative to rule out systemic amyloidosis. The presence of abnormal proteins, such as monoclonal light chains, in the serum and urine detected via immunofixation electrophoresis would indicate systemic amyloidosis. In 
addition, biopsy of an asymptomatic organ or tissue, such as the liver or a less-invasive and highly effective needle aspiration biopsy of subcutaneous adipose tissue, can be stained with Congo red to determine the presence of systemic amyloidosis.

In the case of localized amyloidosis, first line treatment is complete transurethral resection of the amyloid deposits before the mass grows to a significant size capable of hindering bladder or renal function. ${ }^{1}$ Use of intravesical instillation of dimethyl sulfoxide and oral colchicine have been previously reported for treatment of smaller lesions. ${ }^{2}$ Partial cystectomy or cystectomy have also been suggested as second line therapy for large lesions. ${ }^{3}$ Due to the rare nature of this disease, there are no specific guidelines for treatment and follow-up. However, recurrence of localized amyloidosis is common (estimated between 30 - 50\%) and dissemination to systemic amyloidosis remains a possibility. ${ }^{3}$ Therefore, life-long surveillance is often recommended.

Following complete transurethral resection of the amyloid deposits, our patient's lower urinary tract symptoms improved and his hematuria resolved without additional therapies. On annual cystoscopy, our patient remains recurrence free after 5-year follow-up. (Figure 1B).

\section{Conclusion}

Localized primary amyloidosis of the urinary bladder is a rare condition that can closely mimic bladder cancer both clinically and cystoscopically. It is diagnosed by positive Congo red immunostaining of bladder biopsies. First line treatment is transurethral resection and long term follow up is required as recurrence is common. Internal medicine or hematology consultation is paramount to ruling out systemic amyloidosis. Clinicians should maintain a high degree of clinical suspicion as it can be missed on routine histological examination without the use of Congo red staining. 


\section{References}

1. Kobayashi T, Roberts J, Levine J, Degrado J. Primary bladder amyloidosis. Intern Med. 2014;53(21):2511-3.

2. Tolofari S, Ansari A, Knight RJ. A Rare Case of Hematuria; Primary Amyloidosis of the Bladder Neck. Urol Case Rep. 2016 Dec 6;10:48-50.

3. Hosseini A, Ploumidis A, Adding C, Wiklund NP. Radical surgery for treatment of primary localized bladder amyloidosis: could prostate-sparing robot-assisted cystectomy with intracorporeal urinary diversion be an option? Scand J Urol. 2013 Feb;47(1):72-5. 


\section{Figures and Tables}

Fig. 1. Primary amyloidosis of the bladder on cystoscopy at the time of (A) initial cystoscopy after previous resection; and (B) after complete resection.
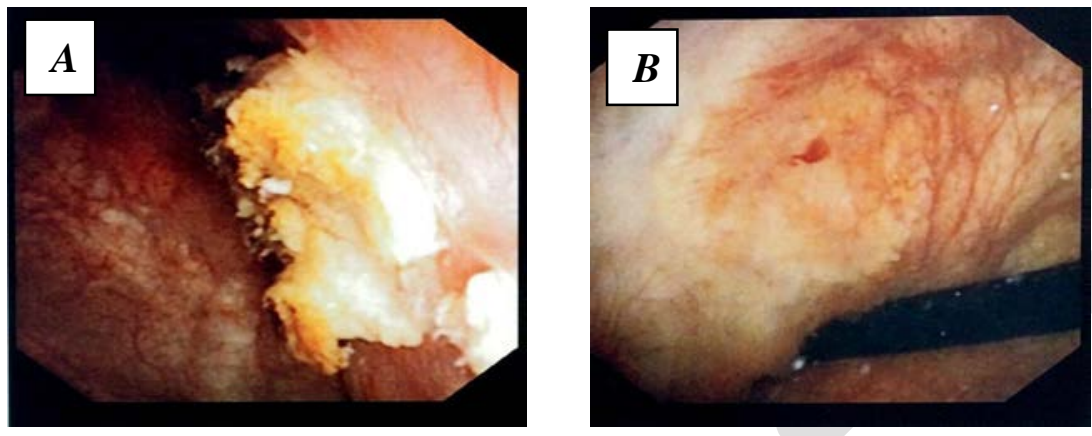

Fig. 2. Histopathology of bladder lesion. (A) Congo red stain (20 x magnification) under unpolarized light (top) and polarized light (bottom) showing amyloid deposits (pink and amorphous) and positive green-apple birefringence under polarized light consistent with amyloidosis of the bladder. (B) Hematoxylin and eosin staining (20 x magnification) showing eosinophilic amorphous deposits.
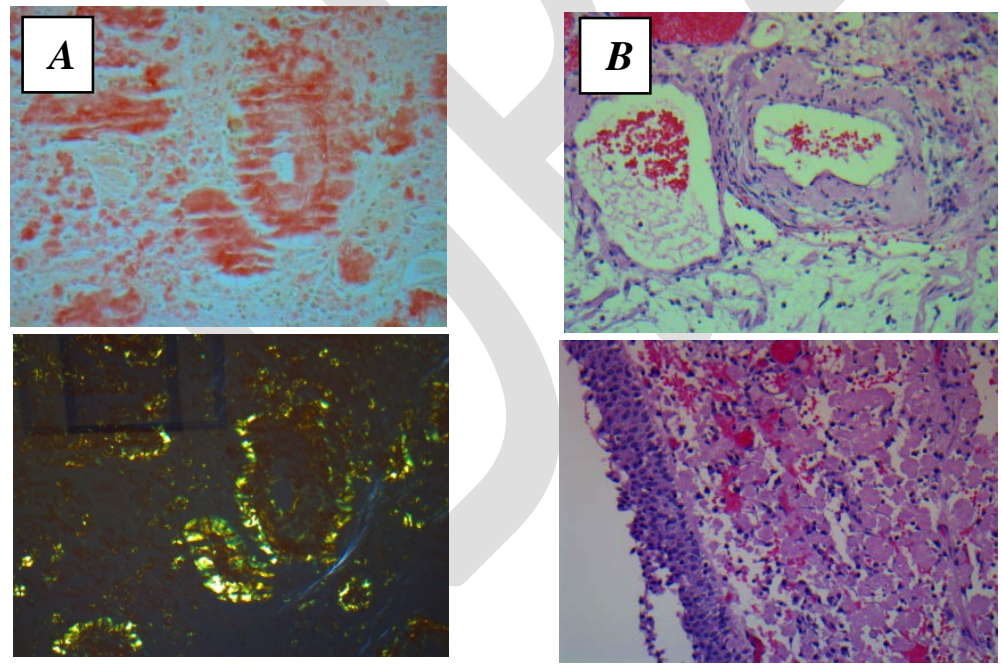